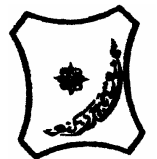

Bayero Journal of Pure and Applied Sciences, 6(1): 118 - 126

Received: January 2013

Accepted: June 2012

ISSN $2006-6996$

\title{
STUDIES ON THE PROPERTIES OF CHITOSAN-STARCH BEADS AND THEIR APPLICATION AS DRUG RELEASE MATERIALS
}

\author{
$*^{1}$ Okolo, P.O., ${ }^{2}$ Akakuru, 0. U., $^{3}$ Osuoji, O.U. and ${ }^{4}$ Jideonwo, A. \\ 1,2,4 Materials Testing Research Centre, Chemistry Department, University of Benin, Benin City, Nigeria. \\ ${ }^{3}$ Department of Physics, University of Benin, Benin City. Nigeria. \\ *Correspondence author: okolopao@yahoo.com
}

\begin{abstract}
In this work, chitosan and blends of chitosan-starch beads were prepared and crosslinked with glutaraldehyde (pentane-1,5-dial). The blends were prepared with 1:1, 2:1 and 3:1 chitosan-starch ratios. Metformin hydrochloride (N,N-dimethylimidodicarbonimidic diamide hydrochloride), a type 2 diabetis drug, was incorporated into them. The materials were subjected to swellability tests and the effects of time and pH were established. Fourier Transformed Infrared Spectrophotometeric (FTIR) analysis was also carried out on the materials and their potentials as drug release systems studied. The results obtained from the FTIR studies showed that the uncrosslinked chitosan-starch bead showed $O-H$ stretching band at $3424 \mathrm{~cm}^{-1}$ and $\mathrm{N}-\mathrm{H}$ band at $1640 \mathrm{~cm}^{-1}$. The chitosan-starch bead also showed a band at $597 \mathrm{~cm}^{-1}$ corresponding to $\mathrm{C}-\mathrm{Cl}$ band, due to the presence of metformin hydrochloride in the bead and its $\mathrm{O}-\mathrm{H}$ stretching band was very strong and broad due to the numerous - $\mathrm{OH}$ groups in starch and chitosan. The results of swelling studies showed that the percent swellability of the polymer matrices increased in both the crosslinked and uncrosslinked beads as the amount of starch in them increased. The uncrosslinked chitosan beads had maximum swelling of $83.74 \%$, while its crosslinked analogue had $47.93 \%$ in acidic medium (pH 2.06) within the range of test period of 60 minutes. The amount of drug released by the 1:1, 2:1 and 3:1 crosslinked chitosan-starch beads (13.90, 13.60, and 12.30mg/L, respectively) was much lower than that of their uncrosslinked analogues (22.50, 20.90, and 16.60mg/L, respectively) after 120 minutes, suggesting that the release of drug is delayed by crosslinking. This suggests that the crosslinked chitosan-starch beads can be used for regulating release of drugs in solid dosages such as beads or tablets.
\end{abstract}

Keywords: chitosan, chitosan-starch beads, drug release, metformin hydrochloride, swelling

\section{INTRODUCTION}

Polymers are macromolecules composed of repeating structural units of monomers connected by covalent chemical bonds. There are many types of polymers and they exist in two broad areas which are natural and synthetic polymers. Natural polymers such as proteins, collagen, silk, keratin, carbohydrates, starch, glycogen, chitosan are widely used materials for conventional applications and even as drug delivery systems. These materials are widely used because they are chemically inert, nontoxic, less expensive, biodegradable, eco-friendly and readily available (Bansal et al., 2011).

Among the naturally occurring polymers, chitosan, starch and their derivatives have been studied by many researchers (Ostrawska-Czubenko et al., 2011). Chitosan is a linear polysaccharide composed of randomly distributed $\beta$-(1-4)-linked Dglucosamine (deacetylated unit) and N-acetyl-Dglucosamine (acetylated unit). Chitosan has also been defined as the deacetylated derivative of chitin which is a water insoluble polymer. ( $\mathrm{N}$-acetyl-Dglucosamine) are found in nature and present in insect exoskeletons, crabs, shrimps, lobsters, and fungal cell walls. It is a natural, biodegradable, biocompatible, bioadhesive polymer, and is gaining attention in the pharmaceutical field for a wide range of drug delivery (Bhumkar and Pokharkar, 2006).
The wide varieties of applications of chitosan are due not only to its excellent biocompatibility, biodegradability, and economic efficiency, but also due to distinct chemical structures with high percentage of primary amino groups $\left(-\mathrm{NH}_{2}\right)$ for easy binding to bio-molecules such as DNAs and proteins (Park et al., 2007).

Blending of chitosan with other polymers such as starch and crosslinking them are both convenient and effective methods of improving the physical and chemical properties of chitosan for practical applications. Crosslinking of chitosan can be achieved by reacting chitosan with epichlorohydrin or ethyleneglycol diglycidyl ether (Ngah et al., 2002), sodium tripolyphosphate (Bhumkar and Pokharkar, 2006), or glutaraldehyde (Ostrawka-Czubenko et al., 2011; Sudha et al., 2012; Kumari and Rani, 2011; Rohindra et al., 2004). Immunization studies carried out on rats using glutaraldehyde-crosslinked chitosan showed promising tolerance by living tissues of the rat muscles (Rohindra et al., 2004), making it a preferred crosslinking agent.

Starch is one of the ubiquitous natural polymers for use as a biopolymer because it is a versatile biodegradable polymer, with immense potential and low price for use in non-food industries (Sudha et al., 2012). 
In view of this, starch has been receiving growing attention since the 1970s. Maize starch and its major components, amylose and amylopectin, are biopolymers which are attractive raw materials for use as components in packaging materials (Sudha et al., 2012). They have been used to produce biodegradable materials to partially or entirely replace plastic polymer films because of their low cost and renewability, as well as their possessing good mechanical properties. However, compared to common synthetic polymers, the biodegradable products based on starch, unfortunately, still revealed many disadvantages which are mainly attributed to the highly hydrophilic character of starch polymer (Sudha et al., 2012).

To overcome the disadvantageous properties of chitosan or starch, the polymer blending technique, which represents a more versatile approach for the development of new materials, is often used. This is because the properties of blends can be varied over a wide range. Relative to a single-component polymeric material, a blend enjoys several degrees of freedom that allow tailoring of the material to meet the requirements suitable for effective drug delivery and even adsorption process (Sudha et al., 2012). Blending of chitosan with starch results in the formation of edible coating with good film forming and mechanical properties (Srisuk and Srikulkit, 2008).

Blended biopolymers can be crosslinked to enhance their properties and broaden their applications. However, the addition of these chemical substances (crosslinking materials) can be limited due to their toxicity (Kumari and Rani, 2011).

The release of drugs from chitosan-based solid dosage depends upon the morphology, size, density, and extent of crosslinking of the particulate system, physico-chemical properties of drug as well as the polymer characteristics such as whether the polymer is hydrophilic or hydrophobic, has gel formation potentials, swelling capacity, muco-adhesive or bioadhesive properties and also depends on the presence of other excepients present in the dosage form (Bansal et al., 2011). Since chitosan does not cause any biological hazard and is inexpensive, it is suitable for use in the preparation of solid dosage forms of commercial drugs (Dutta et al., 2004).

Chitosan and its derivatives in the form of beads have been reported to have effective delivery of some drugs such as insulin, gentamicin sulphate, diclofenac, clarithromicin, cimetidine, famotidine, bovine serum albumin, clozapin, ovalbumin, doxorubicin, metformin hydrochloride, ofloxacin, 5-fluorouracil (Bansal et al., 2011; Kumari and Rani, 2011).

In this study, chitosan-starch beads containing different chitosan-starch ratios, and their crosslinks have been prepared with the incorporation of metformin hydrochloride as a model drug and their swellability and drug release abilities examined. Metformin hydrochloride (N,Ndimethylimidodicarbonimidic diamide hydrochloride) is the first line of drug for the treatment of type 2 diabetis, particularly in overweight and obese people.

\section{MATERIALS AND METHODS}

Glutaraldehyde (pentane-1,5-dial), (25\%) was supplied by Qualikems (India), while metformin hydrochloride was supplied by Ecomed

Pharmaceuticals (Nigeria) as Synformin, sodium hydroxide, acetic acid, sodium hypochlorite, and concentrated hydrochloric acid were of analytical grades from $\mathrm{BDH}$.

\section{Preparation of chitosan from snail shells (Hossein et al., 2008)}

African giant land snail shells (Archarchatina marginata) were collected from a local market in Mbaitoli Local Government Area of Imo State, Nigeria, washed, sun-dried for two weeks and pulverized. The ground shells were later sieved with a mesh sieve $(425 \mu \mathrm{m})$.

The production of chitosan from the ground shells was archieved by the reported methods of Hossein et al., 2008.

A typical process involved the following stages:

(i) deproteinization (ii) decalcification decolouration and (iv) deacetylation.

Deproteinization: The sieved snail shell powder $(250.0 \mathrm{~g})$ was treated with $3.0 \mathrm{~L}$ of $1.2 \mathrm{M} \mathrm{NaOH}$ solution for two and half hours at $75^{\circ} \mathrm{C}$, with stirring at intervals. After the heating process, the solution was allowed to cool, then, the excess $\mathrm{NaOH}$ solution was removed by decantation, followed by washing the powder with deionized water to neutral $\mathrm{pH}$, filtration, and air-drying of the residue (Hossein et al., 2008).

Decalcification: The recovered sample from the deproteinization process $(226.8 \mathrm{~g})$ was placed into $2.72 \mathrm{~L}$ of $0.7 \mathrm{M} \mathrm{HCl}$ solution for 20 minutes. The excess $\mathrm{HCl}$ solution was removed by decantation, followed by washing of the sample to neutral $\mathrm{pH}$ with deionized water, filteration and air-drying (Hossein et al., 2008).

Decolourization: The sample obtained from the decalcification process $(125.4 \mathrm{~g})$ was dispersed in $1.5 \mathrm{~L}$ of $0.3 \%(\mathrm{v} / \mathrm{v})$ solution of $\mathrm{NaOCl}$ (containing $12.5 \%$ available chlorine). The mixture was allowed to stand for 1 hour and the excess $\mathrm{NaOCl}$ removed by decantation, followed by washing to neutral $\mathrm{pH}$ with deionized water and air drying to obtain chitin (95.7g) (Hossein et al., 2008).

Deacetylation of Chitin: The chitin (95.7g) was treated with $1.4 \mathrm{~L}$ of $50 \% \mathrm{NaOH}$ solution (i.e. $12.5 \mathrm{M}$ ) for 20 minutes at about $120^{\circ} \mathrm{C}$. The solution was allowed to cool, then, the excess $\mathrm{NaOH}$ solution was removed by decantation, followed by washing of the sample to neutral $\mathrm{pH}$, filteration with a sintered glass (No. 3) and air drying to obtain chitosan (27.8g) (Hossein et al., 2008).

Preparation of uncrosslinked and crosslinked chitosan-starch beads as drug release systems. Uncrosslinked Beads: Chitosan (1.0g) and metformin hydrochloride $(0.5 \mathrm{~g})$ were mixed together and introduced into $20.0 \mathrm{~cm}^{3}$ of $5.0 \%$ aqueous acetic acid to dissolve. 
Three other portions of $1.0 \mathrm{~g}$ chitosan and $0.5 \mathrm{~g}$ of metformin hydrochloride were also prepared followed by the addition of $0.33 \mathrm{~g}, 0.5 \mathrm{~g}$ and $1.0 \mathrm{~g}$ starch, respectively, and also placed in same concentration of acetic acid to dissolve. This gives $3: 1,2: 1$ and $1: 1$ chitosan-starch ratios, respectively.

The solutions were each stirred for 4 hours to ensure proper homogenization. Each of the solutions were sucked into a $5.0 \mathrm{~cm}^{3}$ syringe and carefully extruded into a $100.0 \mathrm{~cm}^{3}$ solution of $0.5 \mathrm{M} \mathrm{NaOH}$ to coagulate the solutions and form the beads.

The beads were left in the $\mathrm{NaOH}$ solution for 20 minutes with stirring and were thereafter carefully recovered and repeatedly washed with de-ionized water to a neutral pH and air-dried (Kumari and Rani, 2011; Ngah et al., 2002).

Crosslinked beads: Some of the beads were crosslinked by soaking them in $0.1 \%$ glutaraldehyde solution for 24 hours. The crosslinked beads were then recovered, washed repeatedly with de-ionized water to neutral $\mathrm{pH}$, filtered and air-dried. The crosslink density (extent of crosslinking) was also determined (Rohindra et al, 2004).

Characterization of the chitosan and chitosanstarch beads.

FTIR Spectral Analysis:

Infrared transmission spectra of the uncrosslinked chitosan-starch beads were studied using Perkin-Elmer Fourier Transformed Infrared Spectrophotometer (model 2000) (FTIR) in KBr discs from 4000 to $400 \mathrm{~cm}^{-1}$ (Pavia et al, 2001).

\section{Swellability studies}

The swelling ability of the uncrosslinked and crosslinked chitosan and chitosan-starch beads were measured by determining the extent of water uptake, using the methods of Rohindra et al, 2004, the percent water uptake being based on the relationship: $\%$ water uptake $=\underline{\mathrm{W}}_{\underline{\mathrm{s}}}-\mathrm{W}_{\underline{\mathrm{d}}} \times 100$,

$$
W_{d}
$$

where $W_{d}$ and $W_{s}$ are the dry and swollen weights of the materials, respectively. The effect of time on water uptake was carried out by determining \%water uptake of test material at time intervals of $10,20,30,40,50$, and 60 minutes, at fixed $\mathrm{pH}$.

\section{Drug release studies}

The drug release experiments were carried out at $31^{\circ} \mathrm{C}$ in acidic medium (0.1M HCl; pH 2.06). Beads containing the drug (metformin hydrochloride) were placed in the release medium $\left(60 \mathrm{~cm}^{3}\right.$ of $\left.0.1 \mathrm{M} \mathrm{HCl}\right)$ and after 10 minutes intervals, portions of $5 \mathrm{~cm}^{3}$ of the mixture were withdrawn from the beaker for the drug assessment. The withdrawn samples were assessed spectrophotometrically to determine the amount of drug released at $320 \mathrm{~nm}$ where they had maximum absorbance, using a UV-Visible Spectrophotometer (JENWAY 6320D) (Kumari and Rani, 2011).

\section{RESULTS AND DISCUSSION}

Figure 1 shows the spectra of the uncrosslinked chitosan-starch beads. The small sharp but weak band at $3754 \mathrm{~cm}^{-1}$ is attributed to $\mathrm{O}-\mathrm{H}$ stretching, which shows enhanced hydrogen bonding of the $-\mathrm{OH}$ groups. The broad band at $3424 \mathrm{~cm}^{-1}$ is the $\mathrm{O}-\mathrm{H}$ stretching of both starch and chitosan, overlapping the $\mathrm{N}-\mathrm{H}$ stretching vibration of secondary amines in the same region. This band is strong because of the numerous $-\mathrm{OH}$ groups in starch and chitosan. The bands at $2929 \mathrm{~cm}^{-1}$ and $2857 \mathrm{~cm}^{-1}$ represent $-\mathrm{CH}_{2}$ aliphatic groups. The band at $1740 \mathrm{~cm}^{-1}$ corresponds to $\mathrm{C}=\mathrm{O}$ stretching of amide, which may be an evidence of an incomplete deacetylation of the acetyl group in chitin. The doublet at $1640 \mathrm{~cm}^{-1}$ and $1557 \mathrm{~cm}^{-1}$ is attributed to $\mathrm{N}-\mathrm{H}$ bending, which overlaps the $\mathrm{C}=\mathrm{N}$ stretching band in the same region. The strong, broad band at $1411 \mathrm{~cm}^{-1}$ is the $\mathrm{C}-\mathrm{H}$ bending of alkyl groups. The bands at $1140 \mathrm{~cm}^{-1}$ and $1031 \mathrm{~cm}^{-1}$ correspond to $\mathrm{C}-\mathrm{O}-\mathrm{C}$ - glycosidic linkage in the ring. The broad band at $666 \mathrm{~cm}^{-1}$ is attributed to $\mathrm{N}-\mathrm{H}$ bending of primary amines. The band at $597 \mathrm{~cm}^{-1}$ of medium intensity corresponds to the band for $\mathrm{C}-\mathrm{Cl}$, pointing to the incorporated metformin hydrochloride (Pavia et al, 2001).

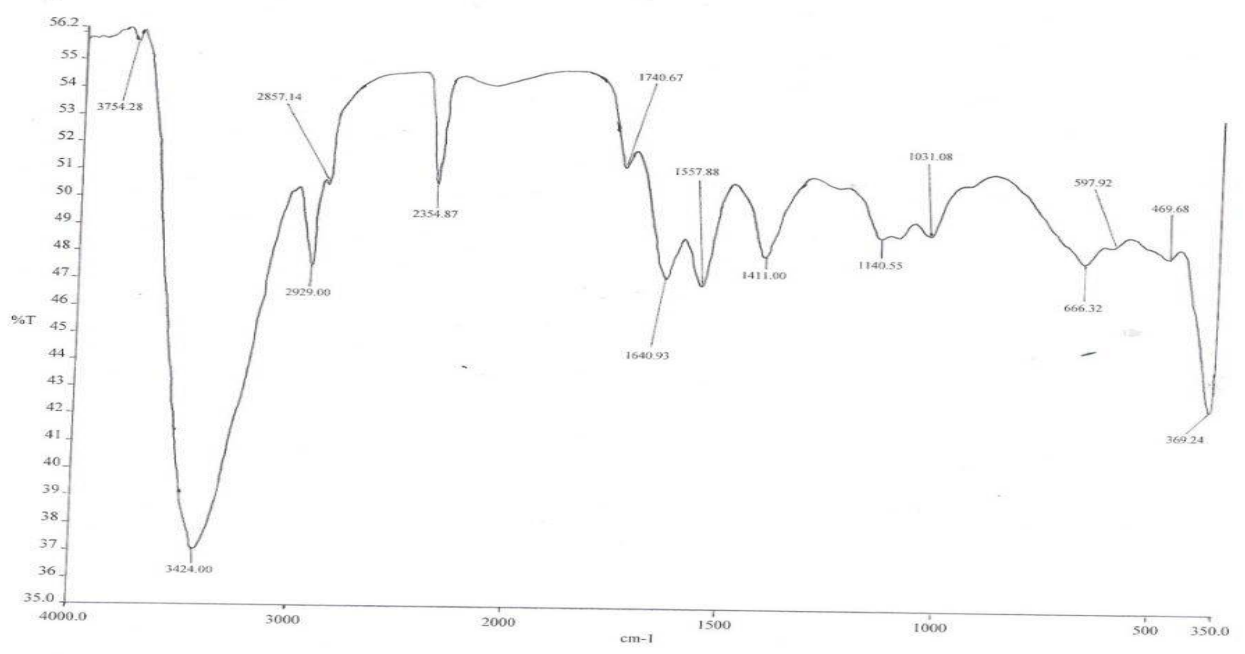

Figure 1: FTIR spectra of undisclosed chitosan - starch beads 
The results of the studies on percent water uptake (swellability) of the chitosan and chitosan-starch beads with time (minutes) in acidic medium ( $\mathrm{pH}$ 2.06) are presented in Figures 2 and 3 (see alsoTables 1 and 2).

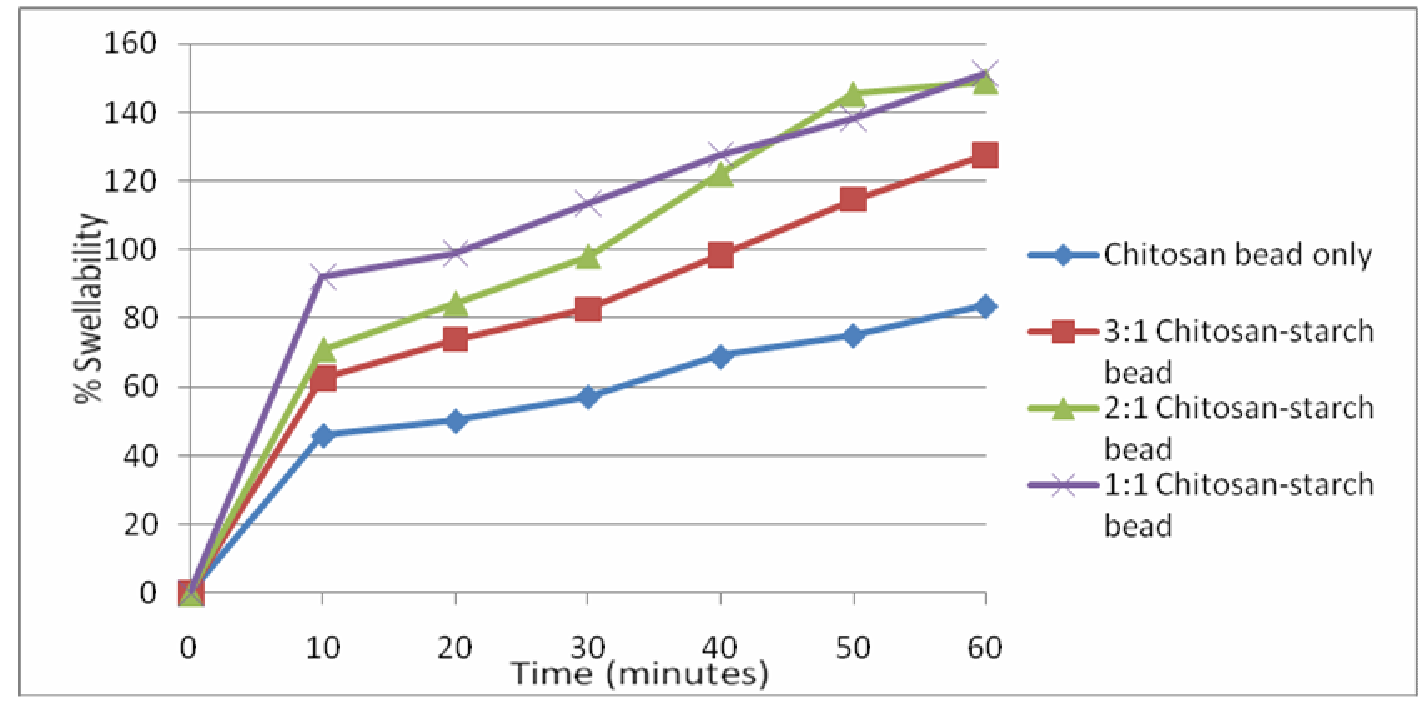

Figure 2: Plot of \% swellability against time (minutes) for uncrosslinked chitosan and chitosanstarch beads in acidic medium (pH 2.06).

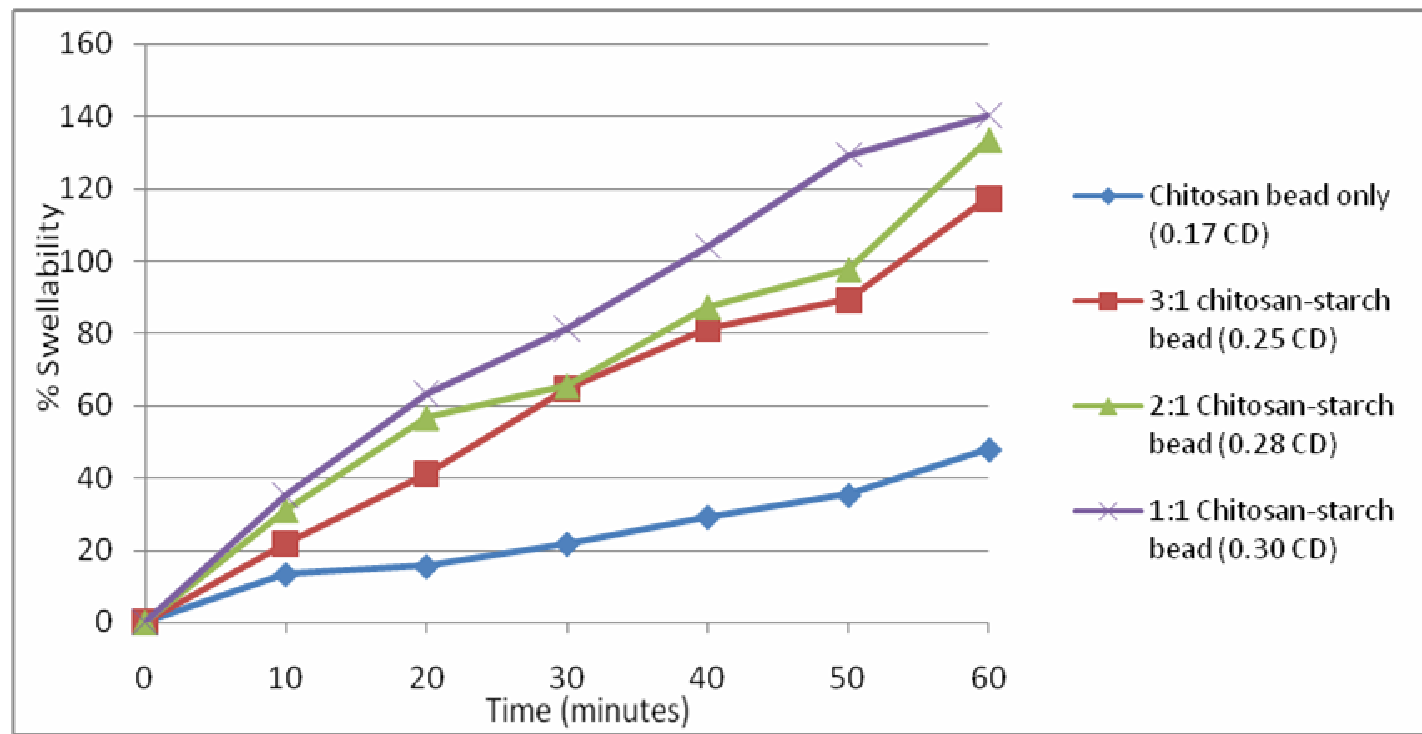

Figure 3: Plot of \% swellability against time (minutes) for glutaraldehyde-crosslinked chitosan and chitosan-starch beads in acidic medium (pH 2.06).

The percent swellability of the uncrosslinked and crosslinked chitosan and chitosan-starch beads increased as time and amount of starch in the beads increases in acidic medium. The increase in swellability followed the order chitosan-starch beads $(1: 1)>(2: 1)>(3: 1)>$ beads with only chitosan. This can be explained based on the fact that, increase in starch composition increased the hydrophilicity of the beads due to the reinforcement with $-\mathrm{OH}$ groups from chitosan. The synergistic effect of $-\mathrm{OH}$ groups (from starch and chitosan) and - $\mathrm{NH}$ groups (from chitosan) may have created enhanced binding sites for water molecules. However, the uncrosslinked chitosan and chitosan-starch beads showed higher percent swellability in acidic medium than their crosslinked counterparts. This is because, crosslinking occurs via the amino groups of chitosan and this is aided by the lone pair of electrons on the nitrogen atoms of the amino groups as shown in Figure 4, reducing the binding sites of the blend for water and therefore affecting swellability.

The results represented in Fig. 2 for the dependence of percent swellability on time for the uncrosslinked chitosan and chitosan-starch beads in acidic medium show that the rate of water uptake (swelling rate) of the uncrosslinked chitosan and chitosan beads is rapid in the first 10 minutes and then becomes almost steady up to 60 minutes, while that for the crosslinked chitosan and chitosan-starch beads represented in Fig. 3 exhibited an almost steady swelling rate from 0 to 60 minutes. 
In general, the swellability of chitosan is affected by three factors:

1. Presence of hydroxyl $(-\mathrm{OH})$ groups in the chitosan chain, which enhance their hydrophilicity.

2. Presence of amino $\left(-\mathrm{NH}_{2}\right)$ groups in the chitosan chain, which gets protonated in water, mostly in acidic medium.

3. Flexibility of the chitosan polymeric matrix, which can allow for easy penetration of solution (Mourya et al., 2010).

The mechanism for the reaction between the amino groups of chitosan and the carbonyl groups of the crosslinking agent, glutaraldehyde as summarized by Ostrawka-Czubenko et al., 2011, which confirms that the amino groups $\left(-\mathrm{NH}_{2}\right)$ of chitosan are the crosslinking sites, is shown in Fig. 4.
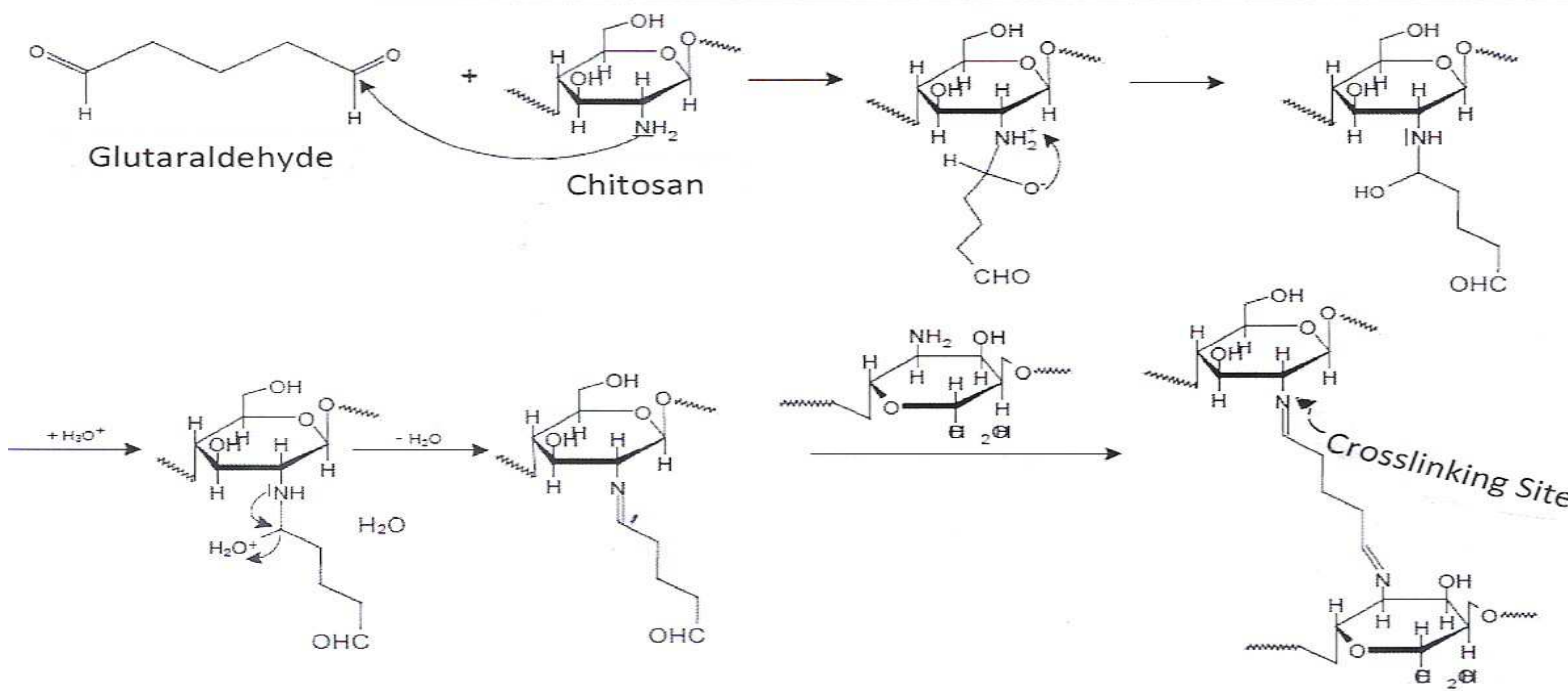

Figure 4: Reaction mechanism for the reaction between the amino group of chitosan and carbonyl groups of glutaraldehyde.

The results of the studies on percent swellability of the chitosan and chitosan-starch beads with time (minutes) in basic medium $(\mathrm{pH}$ 8.03) are presented in Figures 5 and 6 ( see also Tables 3 and 4). The results presented in Figs 5 and 6 show that in

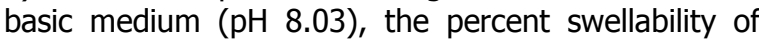
both uncrosslinked and crosslinked chitosan and chitosan-starch beads were lower than in the acidic medium. In basic medium ( $\mathrm{pH} \mathrm{8.03),} \mathrm{the} \mathrm{swelling} \mathrm{that}$ occurred can be said to be mainly driven by solvent diffusion because it is considered that chain penetration by solvent due to protonation of amino groups is absent (Kumari et al., 2011). The lower percent swellability of these beads in basic medium may be attributed to the absence of protonation of the amino groups of the chitosan moiety which would have led to faster intra-hydrogen-bond dissociation (breakage of self association of chitosan and starch) and efficient solvent diffusion (Kumari et al., 2011).

The results represented in Fig. 5 for the dependence of percent swellability on time for the uncrosslinked chitosan and chitosan-starch beads in basic medium show that the rate of increase in percent swellability of the uncrosslinked chitosan and chitosan beads is rapid in the first ten minutes and then becomes almost steady up to 60 minutes, whereas the result represented in Figure 6 for the crosslinked beads show that, the rate of increase in percent swellability of the crosslinked chitosan and chitosan-starch beads was almost steady all through the swelling time of 0 to 60 minutes.

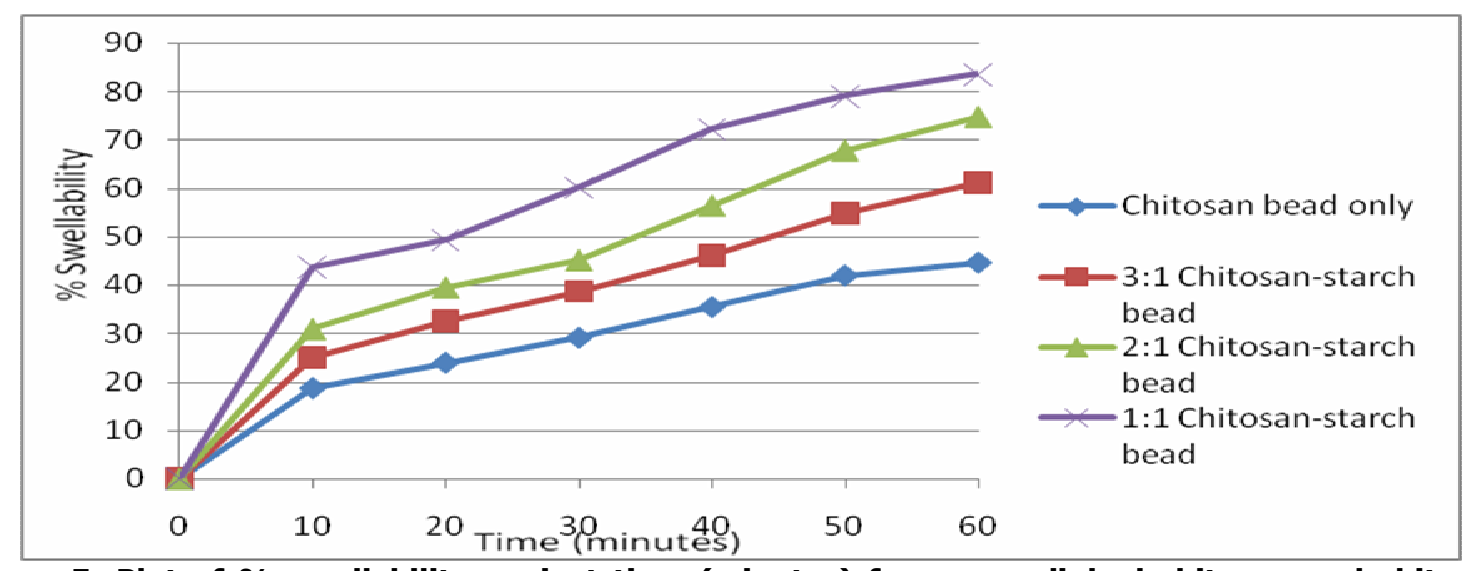

Figure 5: Plot of \% swellability against time (minutes) for uncrosslinked chitosan and chitosanstarch beads in basic medium (pH 8.03). 


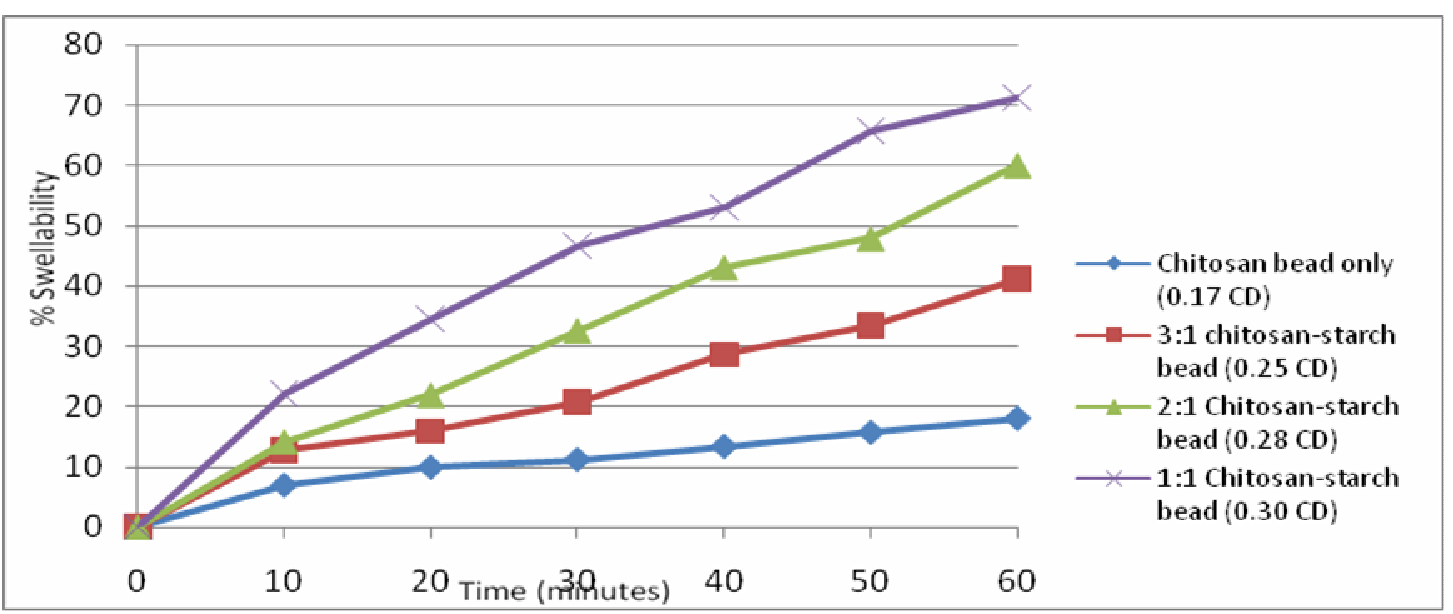

Figure 6: Plot of \% swellability against time (minutes) for glutaraldehyde crosslinked chitosan and chitosan-starch beads in basic medium (pH 8.03).

Figure 7 shows the appearance of the chitosan-starch beads used in the drug release studies. The drug release studies in acidic medium $(0.1 \mathrm{M} \mathrm{HCl}$; $\mathrm{pH} 2.06)$ is important because the target site of activity of the drug used in this study (metformin hydrochloride) is in the human stomach which regularly secretes $\mathrm{HCl}$, making the physiological environment of the stomach to be acidic with a pH range of between 1.0 to 4.5 (Raymond, 1991). The stomach usually maintains a $\mathrm{pH}$ range that may not be too far from $\mathrm{pH} 2$.

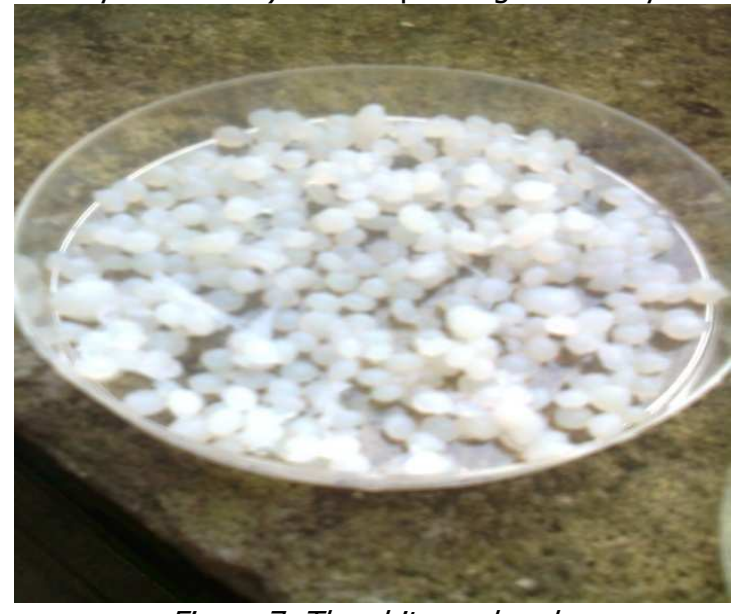

Figure 7: The chitosan beads.

The results of the drug release studies carried out on the uncrosslinked and crosslinked chitosan and chitosan-starch beads in acidic medium (0.1M HCl; $\mathrm{pH} 2.06)$ are shown in Figures 8 and 9 ( see also Tables 5 and 6 ). The uncrosslinked and crosslinked chitosan and chitosan-starch beads showed an increase in the amount of drug released as the time increased as seen in Figs 8 and 9. This increase can also be linked to the amount of starch present in the beads because an increase in the amount of starch increases the number of $-\mathrm{OH}$ groups in the beads, hence increasing hydrophilicity of the beads. This increase in hydrophilicity leads to an increase in the rate at which solvent can penetrate the chitosan-starch bead matrices, causing the beads to swell and release drugs. The results represented in Figs 8 and 9 also show that both uncrosslinked and crosslinked chitosan and chitosan-starch beads showed rapid release of drug in the first 10 minutes of the study and exhibited an almost steady release of drug afterwards. This is because, the mechanism of drug release for materials is that the materials usually swell slowly and their matrices collapse progressively before the release of the drugs in them (Kumari et al., 2011). Comparing the results represented in Figure 8 with those in figure 9 showed that a higher amount of drug was released by the uncrosslinked chitosan and chitosan-starch beads than their crosslinked counterparts. This is obtained by comparing the amount of drug released by the uncrosslinked and crosslinked beads in the first 10 minutes of the study. For example, uncrosslinked chitosan bead released $8.40 \mathrm{mg} / \mathrm{L}$ of drug in the first 10 minutes, while the crosslinked chitosan bead released $5.00 \mathrm{mg} / \mathrm{L}$ of drug. Uncrosslinked 3:1 chitosan-starch bead released $9.50 \mathrm{mg} / \mathrm{L}$ of drug, while the crosslinked $3: 1$ chitosanstarch beads released $7.30 \mathrm{mg} / \mathrm{L}$ of drug in the first 10 minutes. This shows that there is delayed release of drug by the crosslinked chitosan and chitosan-starch beads. This may be because of the compactness of the crosslinked analogue of the beads, leading to less penetration of solvent into their polymer matrix (Kumari et al., 2011). 
Additionally, the unavailability of the amino groups in chitosan as a result of crosslinking, would lead to highly reduced protonation in the medium of study $(0.1 \mathrm{M} \mathrm{HCl} ; \mathrm{pH} 2.06)$. This would reduce the number of $-\mathrm{NH}_{2}$ groups available for solvent absorption. Furthermore, this delay in the release of drug may be due to the immobilization of the chains by the crosslinks and the 'trapping' of drugs within the chitosan and chitosan-starch bead matrices, causing the drugs to be more 'tightly' held and hence slowly released in a delayed manner.

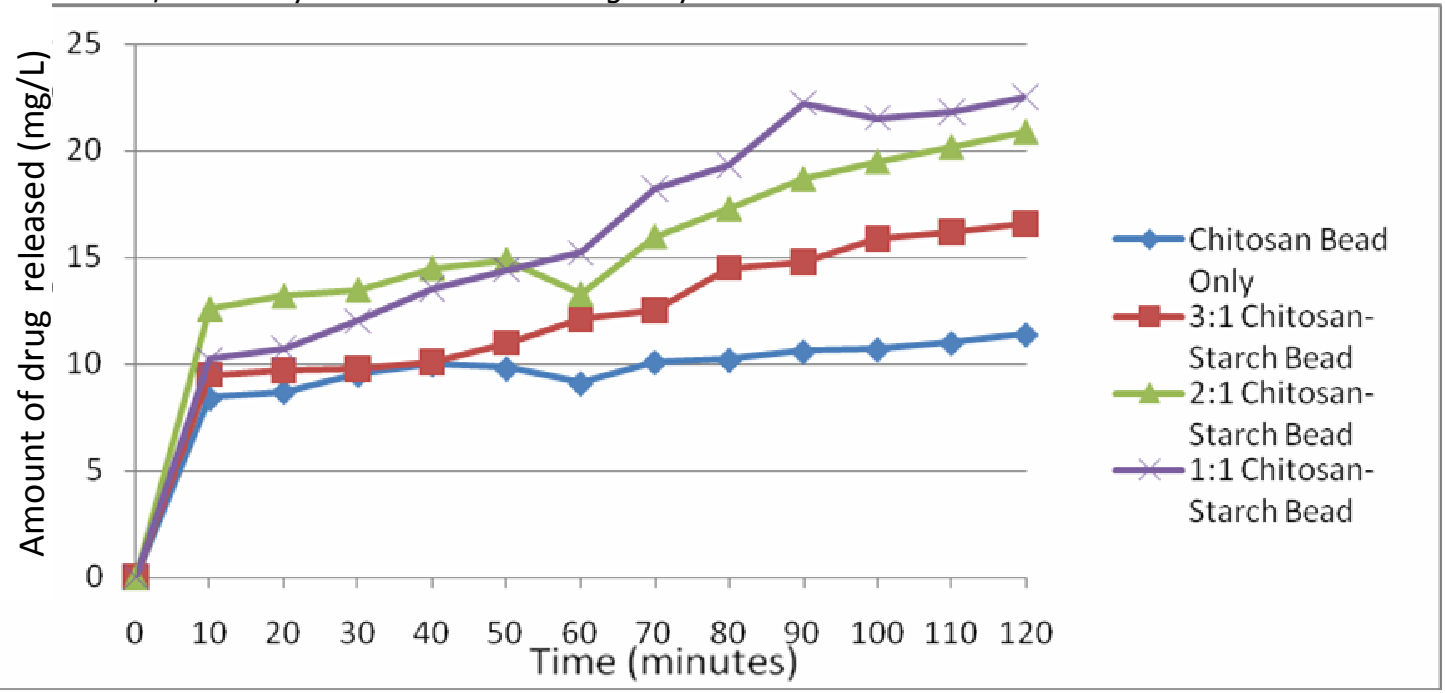

Figure 8: Plot of amount of drug released ( $\mathrm{mg} / \mathrm{L}$ ) against time (minutes) for uncrosslinked chitosan and chitosan-starch beads.

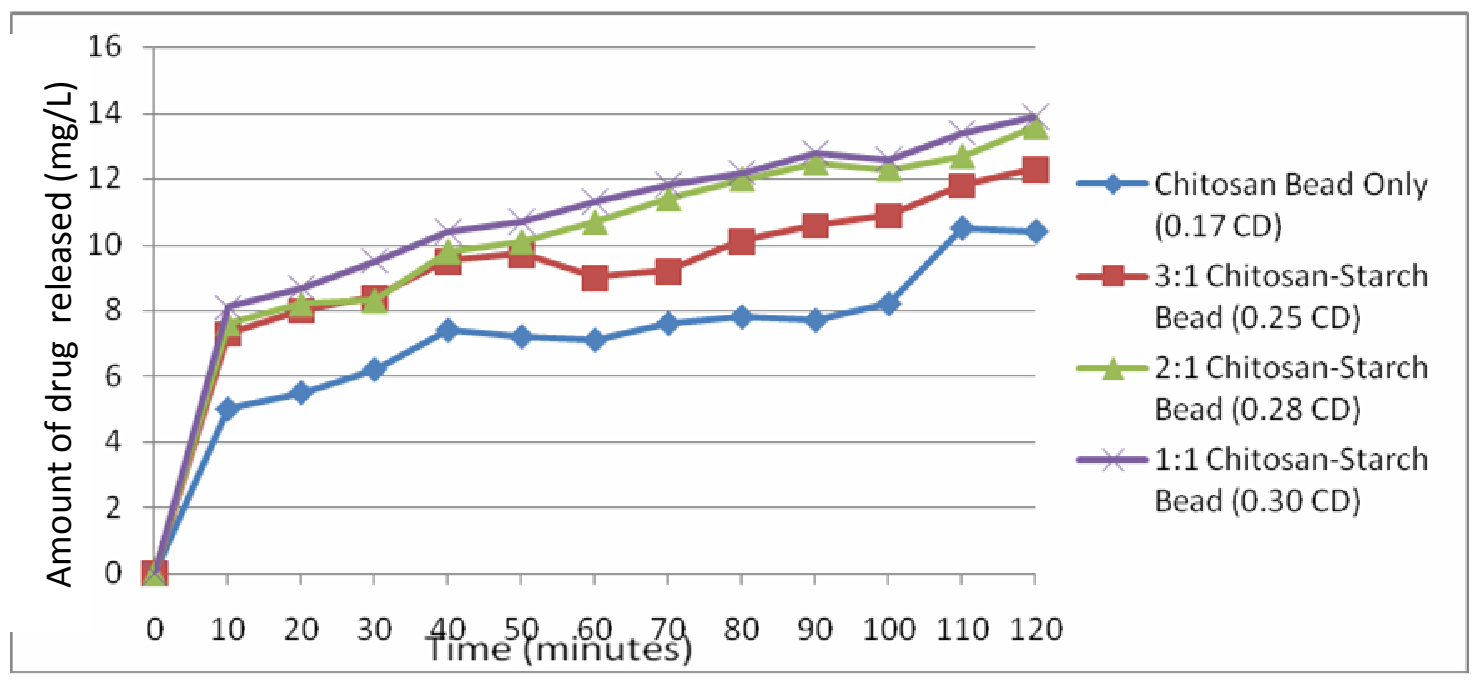

Figure 9: Plot of amount of drug released (mg/L) against time (minutes) for glutaraldehyde crosslinked chitosan and chitosan-starch beads.

\section{CONCLUSION}

From the swelling results, it is clear that the rate of swelling of the chitosan-starch beads was dependent on time and $\mathrm{pH}$. Swellability of the materials containing starch increased as the amount of starch blended with the chitosan increases. Crosslinking clearly caused a slower rate of drug-release in chitosan and chitosan-starch beads. This suggests that by skillfully controlling the factors such as extent of crosslinks, $\mathrm{pH}$ and chitosan-starch ratio, the disintegrants (chitosan and chitosan-starch beads) may be applied for regulating the release of certain drugs, where a controlled rate of drug-release from solid dosage is desirerable. It is common knowledge that metformin hydrochloride is administered one tablet twice daily, with the aim of gradual release from the table. 


\section{REFERENCES}

Bansal, V., Sharma, K.P., Sharma, N., Pal, P.O. and Malviya, R. (2011). Applications of chitosan and chitosan derivatives in drug delivery. Advances in Biological Research, 5(1): 2837.

Bhumkar, D.R. and Pokharkar, V.B. (2006). Studies on effect of $\mathrm{pH}$ on crosslinking of chitosan with sodium tripolyphosphate. A Technical Note. AAPS Pharm. Sci. Tech. 7(2): E50.

Dutta, P.K., Dutta, J., Tripathi, V.S. (2004). Chitin and chitosan chemistry, properties and applications. J. Scientific and Industrial Research, 63:20-31.

Hossein, T., Mehran, M., Seyed, M.R.R., Amir, M.E., Farnood, S.S.J. (2008). Preparation of different chemical processing sequences on the physicochemical and functional properties of the product. J. Molecules, 13: 1263-1274.

Kumari, K. and Rani, U. (2011). Controlled release of metformin hydrochloride through crosslinked blends of chitosan-starch. Advances in Applied Sciences Research, 2(2): 48-54.

ourya, V.K., Inamdar, N.N., Tiwari, A. (2010). Carboxymethyl chitosan and its applications. Advanced materials letters, 1(1): 11-33.

Ngah, W.S.W, Endud, C.S., and Mayanar, R. (2002). Removal of copper (II) ions from aqueous solution onto chitosan and crosslinked chitosan beads. Reactive and Functional Polymers, 50: 181-190.
Ostrawska-Czubenko, J., Pieróg, M. and GierszewskaDrużyńs, M. (2011). State of water in noncrosslinked and crosslinked hydrogel chitosan membranes - DSC studies. Progess on Chemistry and Applicationof Chitin and Its Derivatives, 16:147-156.

Park, I., Cheng, J. and Pisano, A.P. (2007). Low temperature, low pressure nanoimprinting of chitosan as a biomaterial for bionanotechnology applications. Applied Physics Letters, 90: 1-3.

Pavia, D.L., Lampman, G.M. and Kriz, G.S. (2001). Introduction to spectroscopy, $3^{\text {rd }}$ edn. Thompson Learning. Inc. Toronto. Pp 13101.

Raymond, C. (1991). Chemistry, $4^{\text {th }}$ edn. McGraw-Hill, Inc. Princeton. P 565.

Rohindra, D.R., Nand, A.V. and Khurma, I.R. (2004). Swelling properties of chitosan hydrogels. South Pacific Journal of Natural Science. 22(1): 32-35.

Srisuk, S., and Srikulkit, K. (2008). Properties evaluation of some sodium nitrate chitosancotton fabric. Journal of Metals, Materials, and Minerals, 18: 41-45.

Sudha, P.N., Gomathi, T., Govindarajan, C. and Ramasubramanian, S. (2012). Removal of chromium (VI) from aqueous solution using chitosan-starch blend. Der Pharmacia Lettre. 4(1):240-248.

Appendix 1

Table 1: Rate of water uptake (\%) for the uncrosslinked chitosan and uncrosslinked chitosanstarch beads in acidic medium ( $\mathrm{pH}$ 2.06)

\begin{tabular}{|c|c|c|c|c|c|c|}
\hline \multirow[t]{2}{*}{ BEAD TYPE } & \multicolumn{6}{|c|}{ \% SWELLABILITY } \\
\hline & $10 \mathrm{~min}$ & $20 \mathrm{~min}$ & $30 \mathrm{~min}$ & $40 \mathrm{~min}$ & $50 \mathrm{~min}$ & $60 \mathrm{~min}$ \\
\hline Chitosan & 46.03 & 50.27 & 57.32 & 69.01 & 75.14 & 83.74 \\
\hline chitosan-starch (3:1) & 62.64 & 73.91 & 82.74 & 98.52 & 114.73 & 127.61 \\
\hline chitosan-starch (2:1) & 71.08 & 84.52 & 98.21 & 122.16 & 145.41 & 149.03 \\
\hline chitosan-starch (1:1) & 92.16 & 98.87 & 113.64 & 127.88 & 138.23 & 151.47 \\
\hline
\end{tabular}

Table 2: Effect of crosslinking on rate of water uptake for the crosslinked chitosan and crosslinked chitosan-starch beads in acidic medium ( $\mathrm{pH}$ 2.06)

\begin{tabular}{|c|c|c|c|c|c|c|c|}
\hline \multirow{2}{*}{ BEAD TYPE } & \multirow{2}{*}{$\begin{array}{c}\text { CROSSLINK } \\
\text { DENSITY }\end{array}$} & \multicolumn{6}{|c|}{ \% SWELLABILITY } \\
\hline & & $10 \mathrm{~min}$ & $20 \mathrm{~min}$ & $30 \mathrm{~min}$ & $40 \mathrm{~min}$ & $50 \mathrm{~min}$ & $60 \mathrm{~min}$ \\
\hline Chitosan & 0.17 & 13.28 & 15.48 & 21.72 & 29.23 & 35.27 & 47.93 \\
\hline $\begin{array}{c}\text { chitosan-starch } \\
(3: 1)\end{array}$ & 0.25 & 21.72 & 41.18 & 64.67 & 81.23 & 89.36 & 117.51 \\
\hline $\begin{array}{c}\text { chitosan-starch } \\
(2: 1)\end{array}$ & 0.28 & 31.09 & 56.69 & 65.38 & 87.33 & 97.71 & 133.68 \\
\hline $\begin{array}{c}\text { chitosan-starch } \\
(1: 1)\end{array}$ & 0.30 & 35.04 & 63.17 & 81.31 & 104.15 & 129.48 & 140.42 \\
\hline
\end{tabular}

The results in Tables 1 and 2 are represented in Figures 2 and 3 as plots of \% swellability against time (minutes) for uncrosslinked and crosslinked chitosan and chitosan-starch beads respectively. 
Appendix 2

Table 3: Rate of water uptake for the uncrosslinked chitosan and uncrosslinked chitosan-starch beads in basic medium ( $\mathrm{pH} 8.03$ )

\begin{tabular}{ccccccc}
\hline BEAD TYPE & \multicolumn{7}{c}{ \% SWELLABILITY } \\
& 10mins & 20mins & 30mins & 40mins & 50mins & 60mins \\
\hline Chitosan only & 18.68 & 23.91 & 29.04 & 35.38 & 41.87 & 44.53 \\
chitosan-starch (3:1) & 25.09 & 32.53 & 38.77 & 46.19 & 54.92 & 61.28 \\
chitosan-starch (2:1) & 30.89 & 39.52 & 45.16 & 56.41 & 67.82 & 74.73 \\
chitosan-starch (1:1) & 43.70 & 49.34 & 60.18 & 72.25 & 79.11 & 83.62 \\
\hline
\end{tabular}

Table 4: Effect of crosslinking on rate of water uptake for the crosslinked chitosan and chitosan beads in basic medium (pH 8.03)

\begin{tabular}{cccccccc}
\hline BEAD TYPE & CROSSLINK & & \multicolumn{5}{c}{ \% SWELLABILITY } \\
& DENSITY & 10mins & 20mins & 30mins & 40mins & 50mins & 60mins \\
\hline Chitosan only & 0.17 & 06.97 & 09.93 & 11.21 & 13.39 & 15.72 & 18.04 \\
chitosan-starch (3:1) & 0.25 & 12.79 & 15.91 & 20.67 & 28.65 & 33.42 & 41.21 \\
chitosan-starch (2:1) & 0.28 & 14.17 & 21.92 & 32.65 & 43.06 & 47.93 & 60.09 \\
chitosan-starch (1:1) & 0.30 & 22.17 & 34.61 & 46.78 & 53.11 & 65.72 & 71.33 \\
\hline
\end{tabular}

The results in Tables 3 and 4 are represented in Figures 5 and 6 as plots of \% swellability against time (minutes) for uncrosslinked and crosslinked chitosan and chitosan-starch beads respectively.

Appendix 3

Table 5: Dependence of the amount of drug released ( $\mathrm{mg} / \mathrm{L}$ ) on time (minutes) for the uncrosslinked chitosan and chitosan-starch beads in acidic medium

\begin{tabular}{|c|c|c|c|c|}
\hline \multirow[b]{2}{*}{ TIME (mins) } & \multicolumn{4}{|c|}{ AMOUNT OF DRUG RELEASED (mg/L) } \\
\hline & $\begin{array}{c}\text { Chitosan Bead } \\
\text { Only }\end{array}$ & $\begin{array}{c}\text { Chitosan-Starch } \\
(3: 1) \text { Bead }\end{array}$ & $\begin{array}{c}\text { Chitosan-Starch } \\
(2: 1) \text { Bead }\end{array}$ & $\begin{array}{c}\text { Chitosan-Starch } \\
(1: 1) \text { Bead }\end{array}$ \\
\hline 10 & 8.40 & 9.50 & 12.60 & 10.20 \\
\hline 20 & 8.70 & 9.70 & 13.20 & 10.70 \\
\hline 30 & 9.50 & 9.80 & 13.50 & 12.00 \\
\hline 40 & 10.00 & 10.10 & 14.50 & 13.50 \\
\hline 50 & 9.80 & 11.00 & 14.90 & 14.40 \\
\hline 60 & 9.10 & 12.10 & 13.30 & 15.20 \\
\hline 70 & 10.10 & 12.50 & 16.00 & 18.20 \\
\hline 80 & 10.20 & 14.50 & 20.10 & 19.30 \\
\hline 90 & 10.60 & 14.80 & 17.30 & 22.20 \\
\hline 100 & 10.70 & 15.90 & 18.70 & 21.50 \\
\hline 110 & 11.00 & 16.20 & 19.50 & 21.80 \\
\hline 120 & 11.40 & 16.60 & 20.20 & 22.50 \\
\hline
\end{tabular}

Table 6: Dependence of the amount of drug released ( $\mathrm{mg} / \mathrm{L}$ ) on time (minutes) for the crosslinked chitosan and chitosan-starch beads in acidic medium.

\begin{tabular}{ccccc}
\hline TIME (mins) & $\begin{array}{c}\text { Chitosan Bead } \\
\text { Only }\end{array}$ & $\begin{array}{c}\text { AMOUNT OF DRUG RELEASED (mg/L) } \\
\text { Chitosan-Starch } \\
(\mathbf{3 : 1 )} \text { Bead }\end{array}$ & $\begin{array}{c}\text { Chitosan-Starch } \\
(\mathbf{2 : 1}) \text { Bead }\end{array}$ & $\begin{array}{c}\text { Chitosan-Starch } \\
(\mathbf{1 : 1}) \text { Bead }\end{array}$ \\
\hline 10 & 5.00 & 7.30 & 7.60 & 8.10 \\
20 & 5.50 & 8.00 & 8.20 & 8.70 \\
30 & 6.20 & 8.40 & 8.30 & 9.50 \\
40 & 7.40 & 9.50 & 9.80 & 10.40 \\
50 & 7.20 & 9.70 & 10.10 & 10.70 \\
60 & 7.10 & 9.00 & 10.70 & 11.30 \\
70 & 7.60 & 9.20 & 11.40 & 12.20 \\
80 & 7.80 & 10.10 & 12.00 & 12.80 \\
90 & 7.70 & 10.60 & 12.50 & 12.60 \\
100 & 8.20 & 10.90 & 12.30 & 13.40 \\
110 & 10.50 & 11.80 & 12.70 & 13.90 \\
120 & 10.40 & 12.30 & 13.60 & \\
\hline
\end{tabular}

The results in Tables 5 and 6 are represented in Figures 8 and 9 as the amount of drug released (mg/L) against time (minutes) for the uncrosslinked and crosslinked chitosan and chitosan-starch beads respectively. 